\title{
Three-dimensional simulations of ion dynamics in the plasma of an electron cyclotron resonance ion source
}

\author{
V. Mironov and J.P. M. Beijers \\ Kernfysisch Versneller Instituut, University of Groningen, Zernikelaan 25, 9747 AA, Groningen, The Netherlands
}

(Received 14 November 2008; published 1 July 2009)

\begin{abstract}
The ion production in an electron cyclotron resonance ion source (ECRIS) is modeled using a particlein-cell Monte-Carlo-collision code in a three-dimensional geometry. Only the heavy particles (ions and atoms) are tracked, while the electrons are represented using a Maxwell-Boltzmann energy distribution with the electron density determined from the requirement of quasineutrality, and the electron temperature considered as a free parameter. It is found that experimentally observed features of ECRIS plasma are closely reproduced by the code, including the charge-state distributions of extracted ion beams and sputtering patterns inside the source. The isotope anomaly is observed for the mixture of ${ }^{20} \mathrm{Ne}+{ }^{22} \mathrm{Ne}$ isotopes, and a possible explanation is proposed. Finally, the wall-coating effect is treated by modeling the neutralization of ions impinging on the walls of the plasma chamber.
\end{abstract}

DOI: 10.1103/PhysRevSTAB.12.073501

PACS numbers: 29.25.Ni, 52.50.Dg

\section{INTRODUCTION}

The growing demand for intense beams of highly charged ions stimulates further development of an electron cyclotron resonance ion source (ECRIS) [1]. This development is mainly done by following the semiempirical scaling laws [2] -increasing both the confining magnetic fields of the ECRIS trap and the frequency of the microwave radiation that heats the electrons in the source plasma. Extracted ion currents can be increased by applying various special techniques [3] such as gas mixing, wall coating, afterglow, and other effects. The physical mechanisms of these techniques are not yet clearly understood and are a subject of discussion. No generally accepted model has been developed up to now for such important aspects of ECRIS operation as the mechanisms for the ion confinement in the plasma, electron energy distribution, shape of the plasma, etc. Numerical simulations of ECR plasmas can pave the way for a better understanding of the physics of the source and assist in improving the source performance.

We report here on the development of a particle-in-cell Monte-Carlo-collision (PIC-MCC) code designed to model the ion dynamics in the ECRIS plasma. We only consider the dynamics of the heavy particles (ions and atoms), calculating the electron density from the requirement of quasineutrality and taking the parameters of the electron population (the energy distribution function, mean electron energy and spatial variations of these parameters) to be the variable factors. The smallest time scale in the calculations is then defined by the ion movement in the magnetic field, which is in the microsecond range and several orders of magnitude higher than the electron time scale. The largest time scale is determined by the ionization dynamics (around 10 milliseconds). The general idea behind the development is to have a toolkit that enables the estimation of the relative importance of the various processes related to the heavy particle dynamics with respect to the source performance. The code can also be part of a future, more comprehensive code that treats both the electron and ion dynamics in a self-consistent way.

With the present code we could obtain a reasonably good reproduction of the experimentally observed features of ECRIS operation by assuming a spatially uniform Maxwell-Boltzmann electron energy distribution and without any ion confining electric fields in the plasma. Such electric fields are a common assumption in many models that have been developed to describe ECRIS plasma processes [1]. The fields are supposed to be generated by the magnetically confined energetic electrons inside the ECR zone resulting in a localized depression of the positive plasma potential. This "potential dip" confines the ions inside the ECR zone, leading to an exponential dependence of the ion confinement times on their charge states. It should be noted that such a potential dip has not been confirmed experimentally until now; some experiments even seem to contradict a potential dip model $[4,5]$.

\section{CODE DESCRIPTION}

The main approximation made in the code is that the influence of a potential dip on the ion dynamics is negligible. This means that we simulate a quiescent plasma with no gradients of the isotropic electron temperature and without any internal electric fields. The included processes are ion-ion collisions and electron-ion heating in the minimum-B field, as well as the ionization dynamics of ions with different charge states. Neutral particles are tracked in order to take into account the gradients of the gas pressure inside the plasma chamber. Charge-exchange and recombination processes are neglected in our model 
because of their low probability in the simulated plasma conditions.

The calculations are done using the geometry and magnetic fields of the Advanced ECRIS at KVI (KVIAECRIS), which is described in detail elsewhere [6]. The plasma chamber has an inner diameter of $7.6 \mathrm{~cm}$ and a length of $30 \mathrm{~cm}$. The magnetic field has the following properties: the maxima of the solenoidal field are 2.1 and $1.1 \mathrm{~T}$ at the injection and extraction sides; the minimum at the trap center is $0.36 \mathrm{~T}$ and the hexapole field at the radial wall of the source chambers is $0.86 \mathrm{~T}$. The extraction aperture has a diameter of $8 \mathrm{~mm}$. These parameters define the properties of the computational domain of the code.

The solenoid magnetic field of the source is computed using POISSON/SUPERFISH [7], results are stored in a table and imported in the code. Linear interpolation allows calculation of the magnetic field at any position. The hexapolar components of the magnetic field are computed by using the analytical expressions for the Halbach magnetic structure [8]. External electric fields may be incorporated into the calculations when needed. The computational region is meshed using a three-dimensional Cartesian grid. We use a $38 \times 38 \times 64$ mesh in $x, y$, and $z$ directions, respectively, where the $z$ direction is taken along the source axis. We checked that the mesh size is adequate for our purposes.

The ion movement in the magnetic field of the source is tracked using a leapfrog particle mover [9]. The motion of neutral particles is modeled by taking into account scattering and energy losses when they hit the chamber walls.

The collisions between charged particles are computed using the model of Nanbu [10]. In this model a succession of small-angle Coulomb binary collisions is simulated as a single binary collision with a large scattering angle that takes place at each time step. The collision partners are chosen randomly inside a computational cell using the method of Takizuka-Abe [11]. In this way the ion-ion temperature equilibration and ion diffusion processes are taken into account. Ion heating due to electron-ion collisions is modeled by using electrons as the collision partners assuming their isotropic Maxwell-Boltzmann energy distribution. The electron density in a cell is defined by requiring charge neutrality; the electron temperature is considered as a free parameter. The computational time step is chosen to be $10^{-7} \mathrm{sec}$ in order to prevent too large scattering angles in the ion-ion collisions.

More complicated electron energy distributions can be incorporated into the code. In this paper, however, we restrict ourselves to a spatially uniform isotropic Maxwell-Boltzmann distribution.

Knowing the electron density and temperature in a cell, the probability for a heavy particle to increase its charge state $Q$ is calculated using the ionization rates given in Ref. [12]. These rates are tabulated as a function of the electron temperature for all elements from $\mathrm{H}$ to $\mathrm{Zn}(\mathrm{Z}=$
30). Also the contributions of excitation-autoionization processes are included in the rates.

When an ion hits the wall of the plasma chamber it is neutralized and returned into the system to be tracked further. Generally, the scattered atom has an energy that depends on the charge of the impinging ion, the plasma potential, the wall material, and other factors; at this moment we neglect all these effects and randomly pick the energy from a Maxwell-Boltzmann distribution with a temperature of $1 \mathrm{eV}$. The angular distribution of the neutralized particle is assumed to be given by a cosine law [13]. When the neutralized fast atom hits the wall again it loses part of its energy. In the model we set the thermal accommodation coefficient to 0.5 based on experimental data [14] and theoretical estimations [15]. When the energy starts to be comparable to the temperature of the walls, the atom is re-emitted with an energy distribution corresponding to room temperature. In subsequent wall collisions the atom does not change its energy anymore. The effect of the source performance to variations in these parameters will be discussed later in the section that deals with the wallcoating effect. Generally, it was observed in our simulations that the source parameters are not affected significantly by varying the thermal accommodation coefficient and the initial energy of the scattered atoms as long as they are scattered according to the cosine law.

If particles are lost into the extraction aperture, they are injected back as neutrals through an aperture of $1 \mathrm{~cm}$ radius at the injection side of the source, with an energy distribution corresponding to room temperature. The total number of computational particles is always kept at the constant $\left(2 \times 10^{5}\right)$; in this way, the gas flow into the plasma chamber is automatically adjusted to the particle flow out of the source through the extraction aperture. The gas pressure in the chamber is defined by the particle statistical weight and by the total number of neutral particles in the chamber in the stationary conditions.

The calculations are started with equal numbers of atoms and singly charged particles uniformly distributed over the source volume. This ensemble of particles is then allowed to evolve until a stationary condition is reached, which typically takes around $5 \mathrm{msec}$. We checked that the initial distribution of the particles does not influence the stationary solution. In the stationary conditions, the extracted ion currents can be calculated from the flux of ions through the extraction aperture, as well as various other parameters of the ion population.

The code is written in COMPAQ VISUAL FORTRAN and development is done on a personal computer running Windows XP.

\section{RESULTS}

We have used our PIC-MCC code to study the effects of various plasma processes on ion source performance. In the following we discuss results of simulations of the charge- 
state and spatial distributions of ions, the ion confinement times, the electron and ion temperatures, gas mixing and the isotope anomaly, as well as the wall-coating effect. We also briefly discuss the effect of the plasma chamber length on the source performance.

\section{A. Charge-state distributions of ions}

Numerical solutions with ion currents and other parameters close to experimentally observed values can be obtained. This is achieved for gas pressures in the plasma chamber in the range from $10^{-7}$ to $10^{-6} \mathrm{mbar}$, which is within the operational range of typical ECR ion sources. The electron temperature for optimized production of moderately charged ions $\left(\mathrm{Ne}^{6+}, \mathrm{Ar}^{8+}\right)$ is calculated to be around $1 \mathrm{keV}$.

In the simulations we observe that the extracted ion currents as well as their mean charge state increase with increasing gas pressure. This is illustrated in Fig. 1, where neon charge-state distributions are shown for different particle weights between $0.75 \times 10^{8}$ and $2.25 \times 10^{8}$ with a step of $0.25 \times 10^{8}$. This corresponds to gas pressures from $4 \times 10^{-7}$ to $1 \times 10^{-6}$ mbar with an electron temperature $T_{e}$ of $1 \mathrm{keV}$. The arrow in the figure denotes the direction of increasing gas pressure. The total extracted ion current varies from 0.2 to $3.8 \mathrm{~mA}$ for gas pressures in the above-mentioned range. The calculations were stopped at a pressure of $10^{-6}$ mbar because the maximum electron density became comparable to the cutoff density for 14.5 GHz microwaves. The measured distribution of neon ions extracted from the KVI-AECRIS is plotted with open circles. When measuring this distribution, the

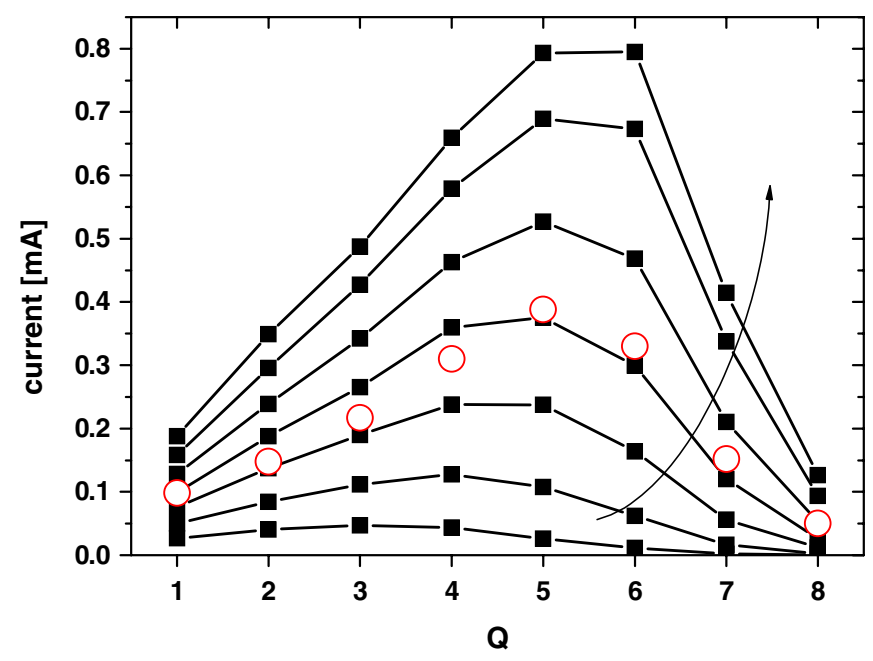

FIG. 1. (Color) Charge-state distributions of the extracted Ne ions as a function of the gas pressure. The arrow shows the direction of increasing gas pressure. Curves correspond to the gas pressures $(3.8,4.8,5.8,6.8,7.6,8.5,9.3) \times 10^{-7}$ mbar. Open circles represent experimentally measured extracted ion currents from the KVI-AECRIS with the source optimized for the maximal $\mathrm{Ne}^{6+}$ current. source was tuned to maximize the output of $\mathrm{Ne}^{6+}$ ions, which means that with increasing gas flow and rf power above the optimized values, the current of $\mathrm{Ne}^{6+}$ did not increase or even decreased.

No saturation with the gas pressure is observed in the simulated currents, which is in contradiction to experiment. It should be noted, however, that an increase in the gas pressure must be accompanied with a corresponding increase in rf power coupled to the plasma to maintain the electron temperature at the same optimal level. In real experimental conditions this is not always possible; the experimentally observed saturation can also be due to an onset of plasma instabilities or other factors.

Figure 1 also shows that in order to ignite a discharge the gas pressure must be above some threshold value-the extracted ion current is very small for neon gas pressures less than $4 \times 10^{-7}$ mbar. This is consistent with operational experiences.

The gas pressure inside the plasma chamber of our source cannot be measured directly during a plasma discharge, but an estimate can be given from the measured value in the vacuum chamber close to the plasma volume; it was around $5 \times 10^{-7}$ mbar when the experimental spectrum of Fig. 1 has been measured. The best correspondence between the simulated and experimental charge-state distribution (CSD) is reached when the particle weights are set to $1.5 \times 10^{8}$, corresponding to an average gas pressure of $6.8 \times 10^{-7} \mathrm{mbar}$. This is close to the experimental values. Given the uncertainties both in the simulations and in experimental conditions, we consider that the agreement between the simulated and measured distributions is good. The same level of agreement is found for all other elements $(\mathrm{N}, \mathrm{O}, \mathrm{Ar})$ used in the simulations. It should be noted that the experimental spectrum was not corrected for the ion losses during beam transport from extraction to the Faraday cup behind the analyzing magnet. Beam transport simulations suggest that the losses can be as high as $30 \%$. We use the simulation conditions with a gas pressure of $7.5 \times 10^{-7}$ mbar as a reference and characterize the plasma parameters in more detail in the following sections, after discussing the selection of the optimal electron temperature.

\section{B. Dependence on the electron temperature}

Dependence of the extracted ion currents on the electron temperature is shown in Fig. 2 for a neon gas pressure of $6.8 \times 10^{-7}$ mbar. A temperature of $1 \mathrm{keV}$ is found to be optimal for maximizing the total extracted ion current and output of neon ions for all charge states except $9+$ and $10+$. Any increase in the electron temperature above this value results in a decreasing source performance. Generally, high electron temperatures are beneficial for the ion confinement in the plasma, since the ion heating rate is inversely proportional to the square root of the electron temperature [16]. For moderately charged neon 


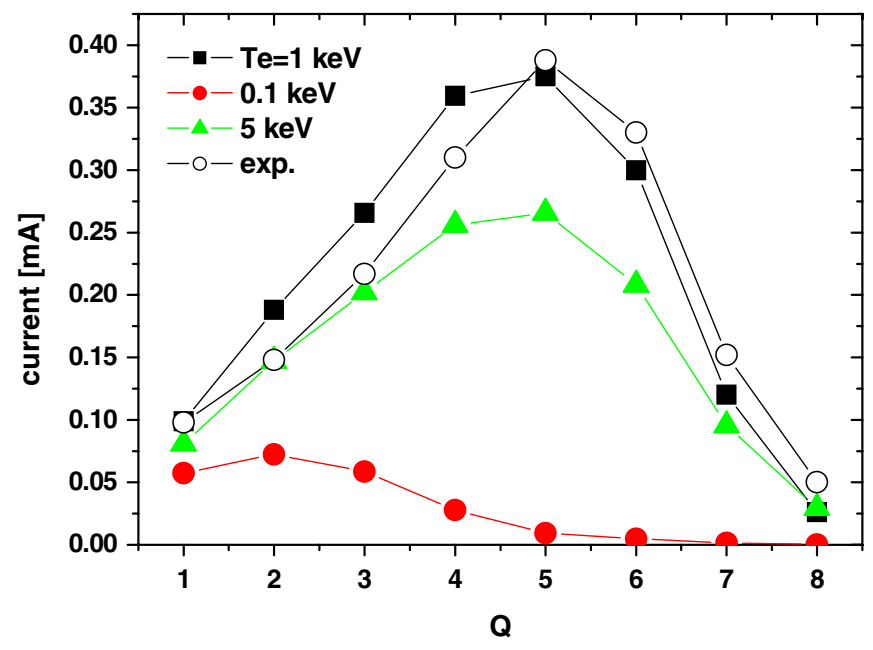

FIG. 2. (Color) Charge-state distributions of extracted neon ions for different electron temperatures. The gas pressure is $6.8 \times$ $10^{-7}$ mbar. Open circles represent experimentally measured extracted ion currents from the KVI-AECRIS.

ions (up to $6+$ ), the ionization rates are decreasing after reaching their maxima for an electron temperature around $1 \mathrm{keV}$. At an electron temperature of $5 \mathrm{keV}$, the rate of $\mathrm{Ne}^{1+}$ production drops by $30 \%$ compared to the value at a temperature of $1 \mathrm{keV}$. The rates for the highest charge states $(8+, 9+)$ increase with this variation of electron temperature by a factor of more than 3 , and the total content of the highest charge states in the plasma goes slightly up even with the decreased content of their predecessors. The drop in the production rates of moderately charged ions has a more pronounced effect than the decrease in the ion heating rate. If the electron temperature is less than $500 \mathrm{eV}$, the ion output decreases both due to a drop in the ionization rates and an increase in the heating rate.

Many experiments have demonstrated the existence of a cold electron population in ECRIS plasma, mainly outside of the resonance zone. We have studied the influence of cold electrons $(1-50 \mathrm{eV})$ with our code. It was found that if these electrons are located in the regions that are not magnetically connected to the ECR zone, their presence does not change the results significantly, because the plasma is strongly localized along the source axis as will be described in the next section.

\section{Spatial distributions of the ions}

Large gradients of the ion densities in the radial direction are observed. A projection of the total ion density on the $x-y$ plane, averaged along the source axis, is shown in Fig. 3. The maximum density is $3.2 \times 10^{11} \mathrm{~cm}^{-3}$ for the reference plasma conditions. The characteristic six-arm star is due to the hexapole magnetic field. The ion density is strongly concentrated on the source axis, and the higher the charge state of the ions, the more peaked their spatial distribution is. This is illustrated in Fig. 4, where the

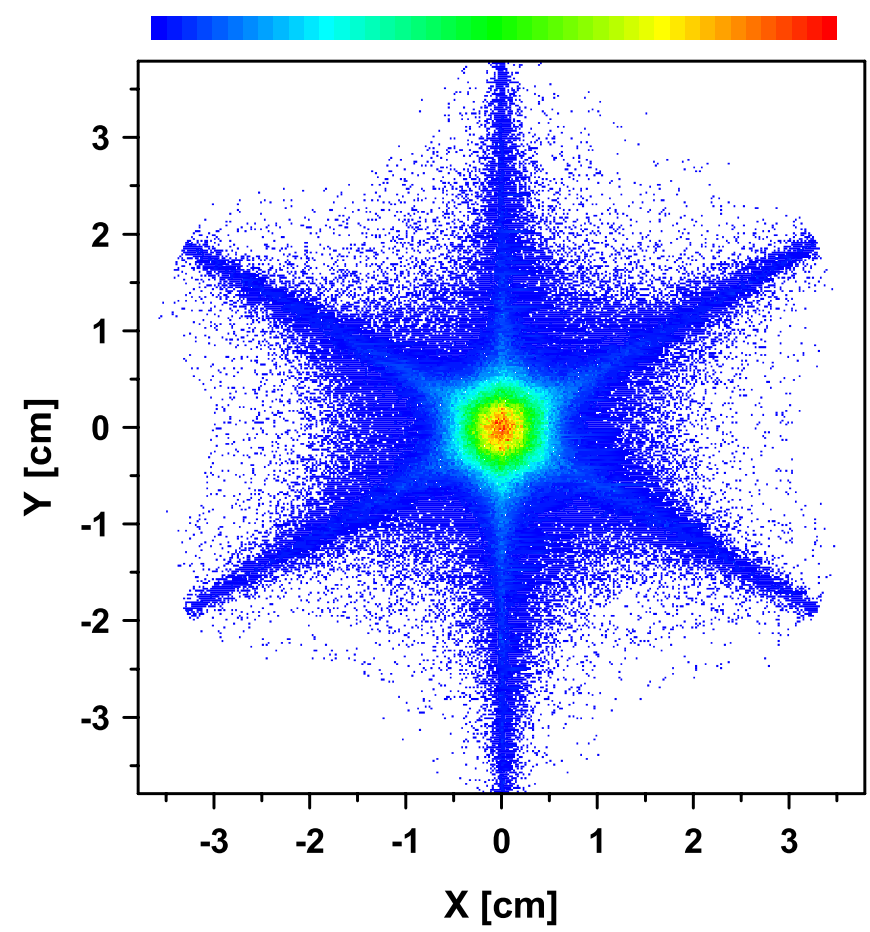

FIG. 3. (Color) The $x-y$ profile of the ion density. The maximum density at the axis of the source is $3.2 \times 10^{11} \mathrm{~cm}^{-3}$.

profiles of the electron density and of the mean ion charge state are shown along the $x$ axis (between the star arms). The values are averaged along the $z$ axis. The electron density reaches a maximum value of $1.1 \times 10^{12} \mathrm{~cm}^{-3}$ on the axis, and the plasma diameter is around $1 \mathrm{~cm}$. The mean charge state increases from $1+$ at the plasma periphery to $4+$ at the source axis.

The profile of the electron density along the source axis is shown in Fig. 5. The density is gradually decreasing when approaching the extraction and injection sides of the source. The mean charge state of the ions is moderately peaked at the plasma center.

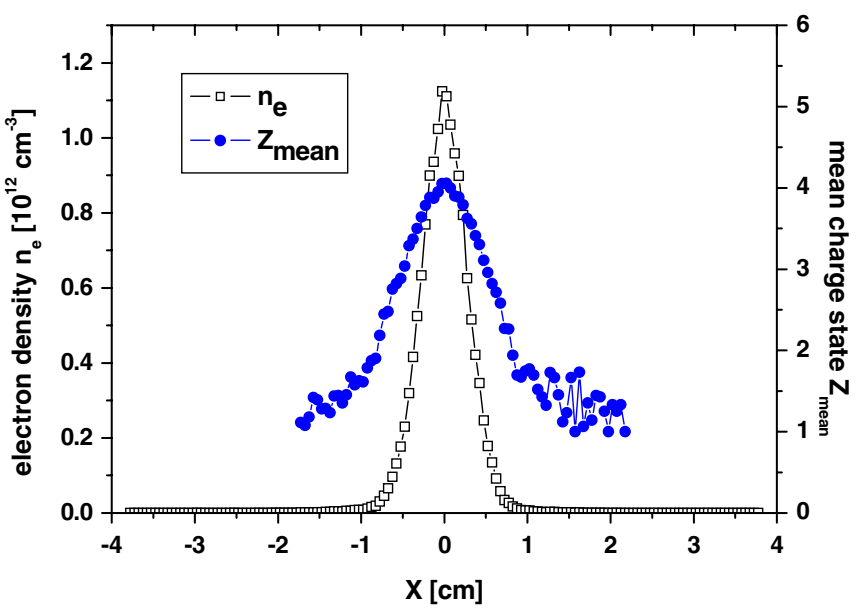

FIG. 4. (Color) Electron density (left scale) and mean ion charge state (right scale) at $y=0$ (between the star arms) as a function of $x$. The values are averaged in the $z$ direction. 


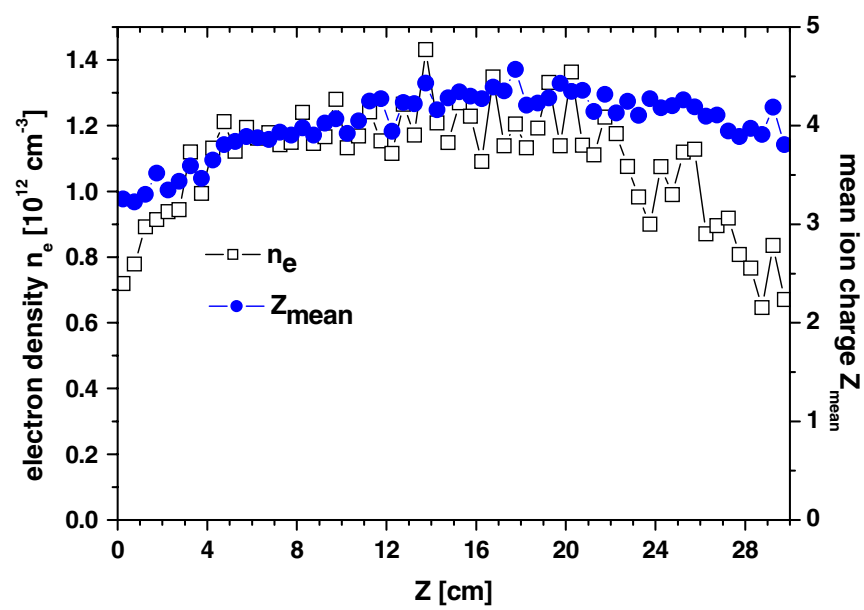

FIG. 5. (Color) Electron density (left scale) and mean ion charge state (right scale) at the axis of the source as a function of $z$.

A strong "ion pumping" effect results in variations of the gas density inside the source. At the source axis, the neutral density is around $50 \%$ of the average value. This drop in the gas density drastically reduces the ion output of the source in comparison with the situation when the neutral particles are uniformly distributed in the chamber. Experimentally, gas starvation is generally considered to be beneficial for the production of the highest charge states; however, in our model the higher the gas pressure, the higher is the output of the highest charge states. This shows the model limitations.

The positions where the ions are hitting the plasma electrode are shown in Fig. 6. At the injection side of the plasma chamber, the pattern is basically the same with the three-arm "star" rotated by $180^{\circ}$; it is more compact because of the higher magnetic field. The same tendency as with the simulated spatial distribution of the ions inside the plasma is observed-ions with higher charge states hit the plasma electrode at smaller radii. Mean radial sizes of the beam profiles at the plasma electrode are shown in Fig. 7 for the reference calculations for neon. The localization of high charge states closer to the source axis is supported by experimental observations [17] of the ion beam normalized emittances as a function of the charge state for a source that is fairly similar to ours. This is also observed for other ECR sources [18] and seems to be a general tendency.

A specific feature of the pattern shown in Fig. 6 is that the ion flux into the extraction aperture has a pronounced triangular symmetry. This results in a triangular shape of the extracted ion beams, which is confirmed by beam profile measurements [19]. Excluding the extraction aperture, the most pronounced ion flux to the plasma electrode occurs along the axes of symmetry in the star arms with a relatively broad halo of predominately singly charged ions. This is in good agreement with the experimental observations of narrow sputtering lines along the star arm axes.

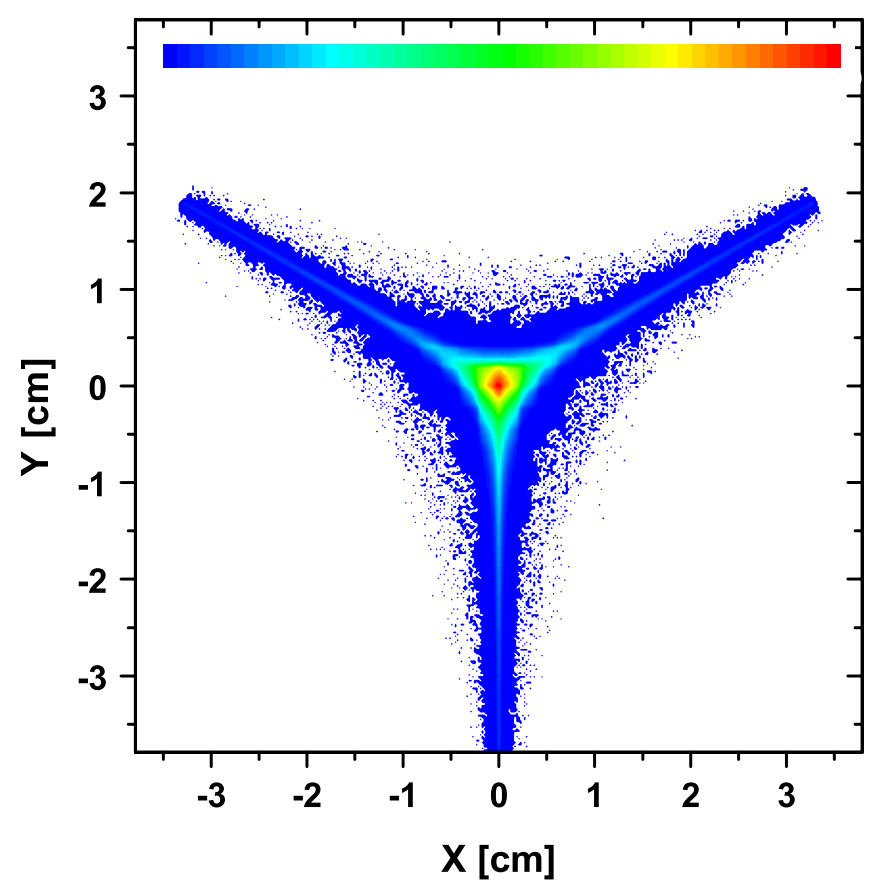

FIG. 6. (Color) Spatial distribution of the lost neon ions at the plasma electrode.

Intense sputtering of the electrode at the injection side of the source occurs at the center, again consistent with the simulations.

The effective electron density depends on the ion charge state as shown in Fig. 7. The effective density is defined in the following way: for each ion with a specific charge state $Q$ we compute the average electron density along the path of this ion from the moment when the ion is ionized into the state $Q$ until the moment when the ion is lost at the plasma electrode. Then, these values are averaged over the whole ion population with charge state $Q$. For the reference neon conditions, the effective electron density is

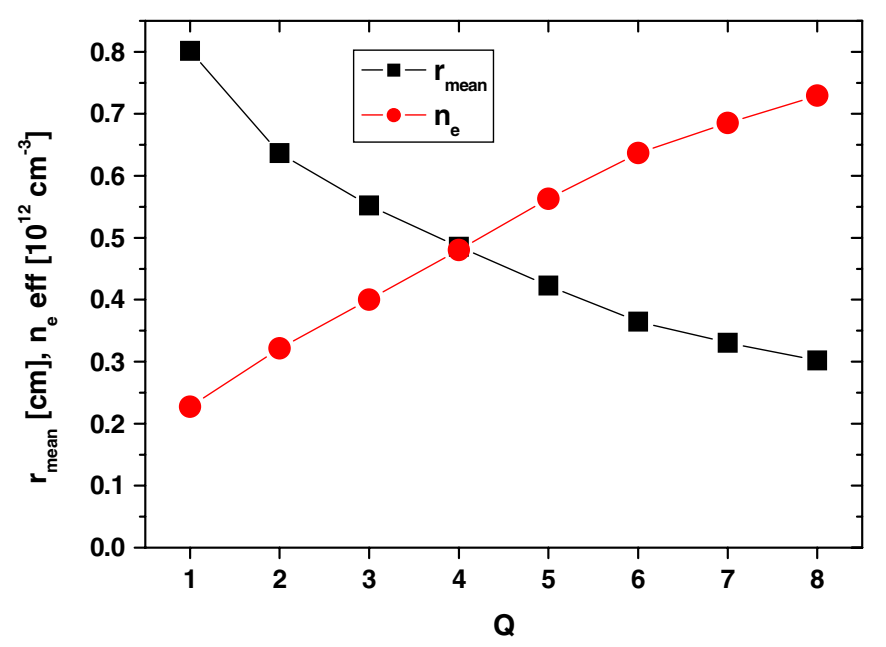

FIG. 7. (Color) Mean radius of the ion beam profile at the plasma electrode (squares) and the effective electron density (circles) for neon ions as a function of charge state. 
$0.7 \times 10^{12} \mathrm{~cm}^{-3}$ for the high charge state $(8+)$, which is a factor of 3 higher than for the $\mathrm{Ne}^{1+}$ ions.

\section{Ion temperatures}

Temperatures of the ions inside the plasma are calculated to be around $0.25 \mathrm{eV}$ (for neon in the reference conditions). The singly charged ions $(0.15 \mathrm{eV})$ are much colder than the ions with higher charge states; the temperature of these highly charged ions slowly increases with their charge state. No experimental data on the ion temperatures in the ECRIS plasma are available, though there are indications that they are in the $1 \mathrm{eV}$ range [20]. Experimentally observed relatively large energy spreads in the extracted ion beams are sometimes interpreted in terms of large ion temperatures $(5-10 \mathrm{eV})$ inside the plasma [21]. Our calculations do not support such interpretations.

While the velocity distributions for the ions inside the plasma can be well fitted with a Maxwell-Boltzmann distribution, the distributions of the lost ions are anisotropic. Significant differences are seen for the velocity distributions along the source axis (along the magnetic field lines) compared to the perpendicular direction. This is illustrated in Fig. 8 for neon ions. Acceleration of the ions along the source axis is caused by the ion pressure gradient. The resulting mean energy of the extracted ions is a factor of 1.5 higher than the mean energy of the highly charged ions inside the plasma, i.e., 0.55 and $0.35 \mathrm{eV}$, respectively. As a result of this acceleration the confined ion population is cooled.

\section{E. Ion confinement times}

The residence times of the extracted ions with different charge states can be calculated by measuring the time intervals between the moment when a singly charged ion is created from a neutral and the moment when this ion, being ionized to the charge state $Q$, is lost at the extraction

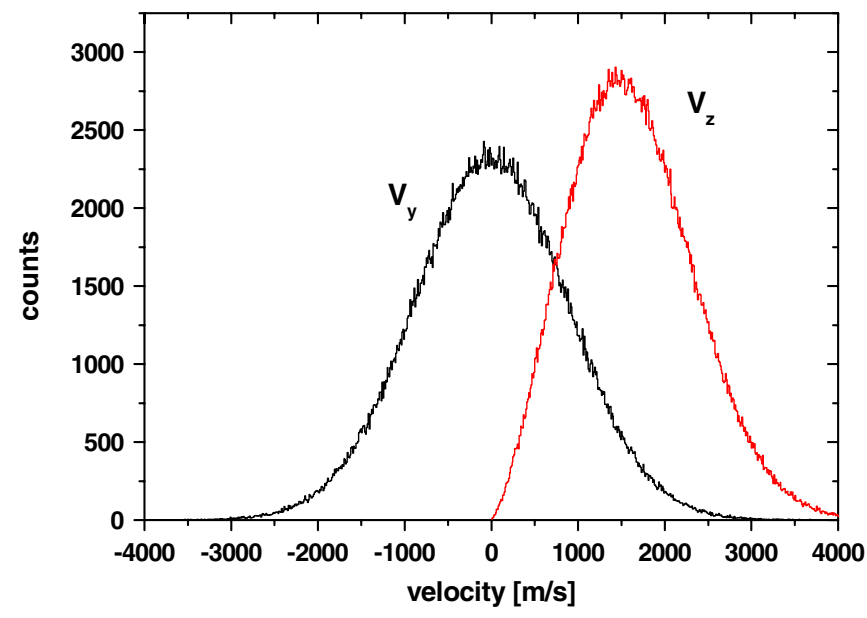

FIG. 8. (Color) Velocity distributions in $y$ and $z$ directions of the neon ions that are lost at the plasma electrode. aperture. These first-passage-time distributions (FPTD) are presented in Fig. 9 for extracted neon ions in the charge states from $1+$ to $8+$, together with the cumulative distribution for all charge states. The higher the charge state, the longer is its mean time of residence in the plasma. Reversing this point of view, ions with longer residence times have better chances to be ionized to higher charge states. The tail of the cumulative FPTD can be well fitted with an exponential decay curve; the decay constant is around $0.65 \mathrm{~ms}$ for the reference conditions.

An experimental method to observe the FPTD of Fig. 9 is, e.g., a pulsed injection of metal atoms into the ECRIS plasma followed by measuring the arrival times of the extracted and charge-analyzed ions.

The mean confinement time for ions can be defined as the ratio between the total number of ions with given charge state that stay in the plasma and the flux of these ions to the plasma chamber walls. The mean confinement times as a function of the ion charge state are shown in Fig. 10 for neon ions. It is close to linear for the lower charge states and then saturates at a level of around $0.62 \mathrm{~ms}$. The same dependence is also observed for other elements, but each element saturates at a different level, e.g., for argon ions the confinement time saturates at $0.8 \mathrm{~ms}$. This behavior agrees well with measurements of the ion confinement times in an argon ECRIS plasma [4]. In these measurements, ion densities at the source axis were determined from the intensity of the characteristic $\mathrm{x}$-ray emission of the highly charged argon ions, and the ratio between these densities and the extracted ion fluxes was defined as the ion confinement time. A weak linear dependence was observed for the charge states of argon

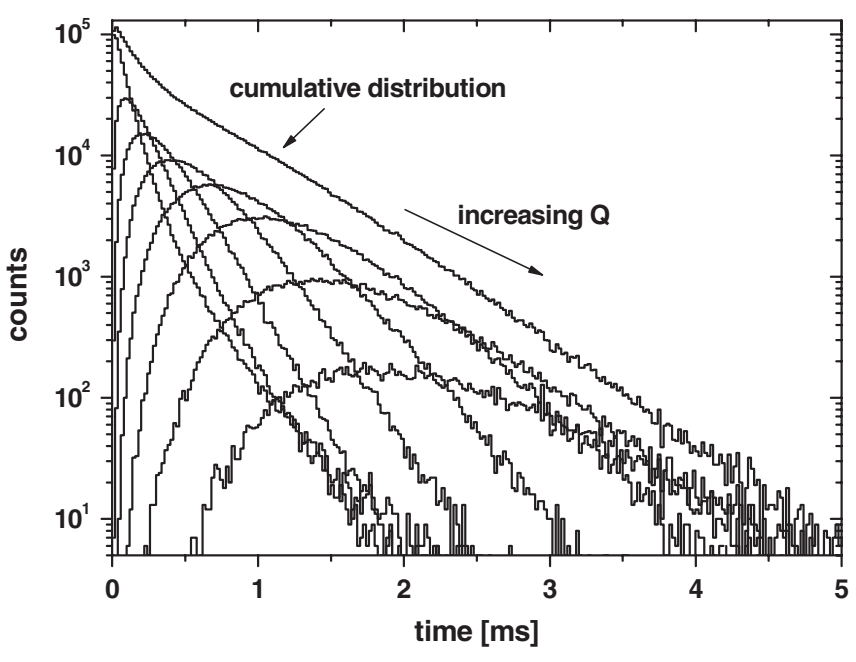

FIG. 9. Distribution of residence times inside the plasma for the different charge states of extracted neon ions from $1+$ to $8+$ and the cumulative distribution. The residence times are determined from the moment of neutral atom ionization until the moment when the ion with the given charge state is lost in the extraction aperture. 


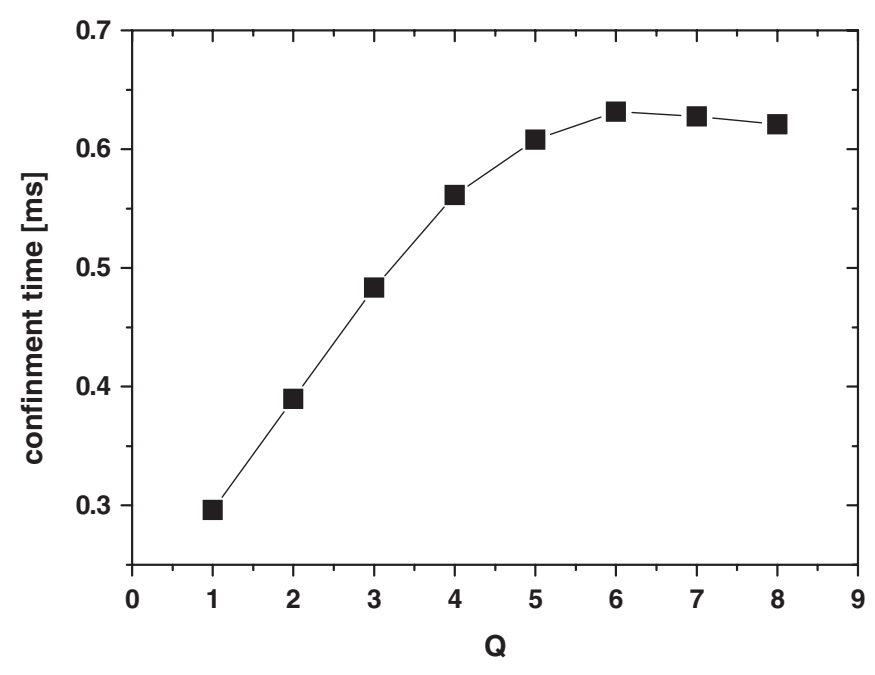

FIG. 10. Dependence of the mean confinement time on the ion charge state for neon. The plotted confinement times are based on losses of ions to the walls and by ionization to higher charge states.

ions ranging from $9+$ to $16+$, and it was nearly constant for a plasma optimized for relatively low charge states.

It should be noted that the distributions for the specific charge states of Figs. 9 and 10 reflect both the losses of the ions to the chamber walls and losses due to ionization into the higher charge states. For the highest charge states, the losses to the chamber walls are dominant because of the low ionization probability. The cumulative distribution of Fig. 9 does not include the ionization losses and relates to the wall losses only. The decay constant of the cumulative distribution is close to the value of the mean confinement time for the highest charge states in Fig. 10. We take this value as the ion confinement time for the plasma in our reference conditions.

The ion confinement time in the so-called gas-dynamics mode, i.e., in the limit of a zero potential dip, is given [22] by $\tau_{c}=R L / v_{i}$, where $R$ is the magnetic mirror ratio, $L$ is the characteristic length of the plasma and $v_{i}$ is thermal velocity of ions. The ion confinement time does not depend on the ion charge state. The mirror ratio along the source axis for the extraction side of our source is equal to 3 , the plasma half-length $L$ is $15 \mathrm{~cm}$ and the mean velocity is $1.6 \times 10^{5} \mathrm{~cm} / \mathrm{sec}$ for the $\mathrm{Ne}^{6+}$ ions in the reference condition, corresponding to a thermal velocity of $1.0 \times$ $10^{5} \mathrm{~cm} / \mathrm{sec}$; the resulting estimate for the confinement time is $0.45 \mathrm{~ms}$, in reasonable agreement with our simulations. The gas-dynamics confinement defines the scale of plasma production in our simulations.

\section{F. Gas-mixing and isotope anomaly}

After having characterized the ion output from the ECRIS plasma in the reference conditions, we now present some results obtained by varying specific source parameters. First, it was checked whether the isotope anomaly [3]

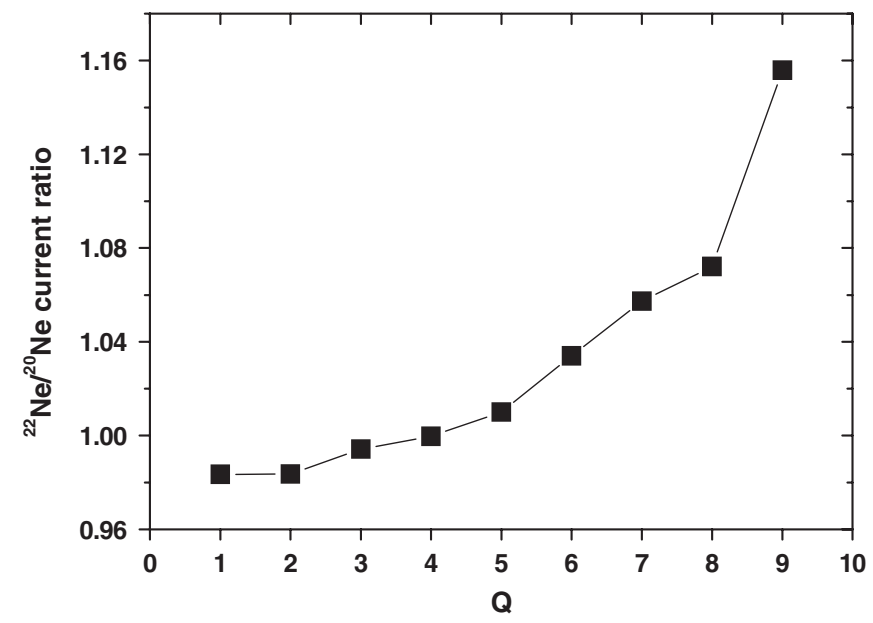

FIG. 11. Ratio between the extracted currents of ${ }^{22} \mathrm{Ne}$ and ${ }^{20} \mathrm{Ne}$ ions as a function of the charge state.

can be reproduced by the model. This was done by running simulations starting with an equal mixture of ${ }^{20} \mathrm{Ne}$ and ${ }^{22} \mathrm{Ne}$ isotopes and keeping the isotope fluxes into the source equal during the run.

A pronounced effect is observed for the ratio of extracted currents for both isotopes as a function of the ion charge state, see Fig. 11. The current of ${ }^{22} \mathrm{Ne}^{9+}$ is $15 \%$ higher than the ${ }^{20} \mathrm{Ne}^{9+}$ current, while the currents of the low charge states for the heavier isotope are 5\% smaller. The same effect for neon isotopes is also experimentally observed in our source.

Differences in the residence times for the isotopes are observed: the slopes of the FPTD tail that are defined as shown in Fig. 9 are $0.67 \mathrm{~ms}$ for ${ }^{20} \mathrm{Ne}$ and $0.69 \mathrm{~ms}$ for ${ }^{22} \mathrm{Ne}$ ions for the simulated plasma. The ratio of these values scales approximately as the square root of the mass ratio and corresponds to the ratio between the mean velocities of the ions. The heavier isotopes stay in the plasma for a longer time because of their lower mean velocities.

Beam radii at the extraction plane and the effective electron densities for the ${ }^{20} \mathrm{Ne}$ and ${ }^{22} \mathrm{Ne}$ isotopes are shown in Fig. 12. Noticeably different spatial distributions are observed for the isotopes-beam profiles of the heavier ${ }^{22} \mathrm{Ne}$ ions at the plasma electrode are systematically more compact than for the lighter isotope. This can be caused by the slower outward diffusion of the heavier particles in the radial direction. The reason for this is that the diffusion rate is determined by the ion thermal velocities and not by the Larmor radius when the ion-ion collision rate is much higher than the cyclotron frequency. Taking into account the large radial gradients of the plasma density, the heavier isotope stays for a longer time in the regions with larger mean electron density (up to $2 \%$ ) when it diffuses through the plasma, compared with the lighter isotope. Combined together, these two factors lead to a more effective production and extraction of the higher charge states for the heavier isotopes. 


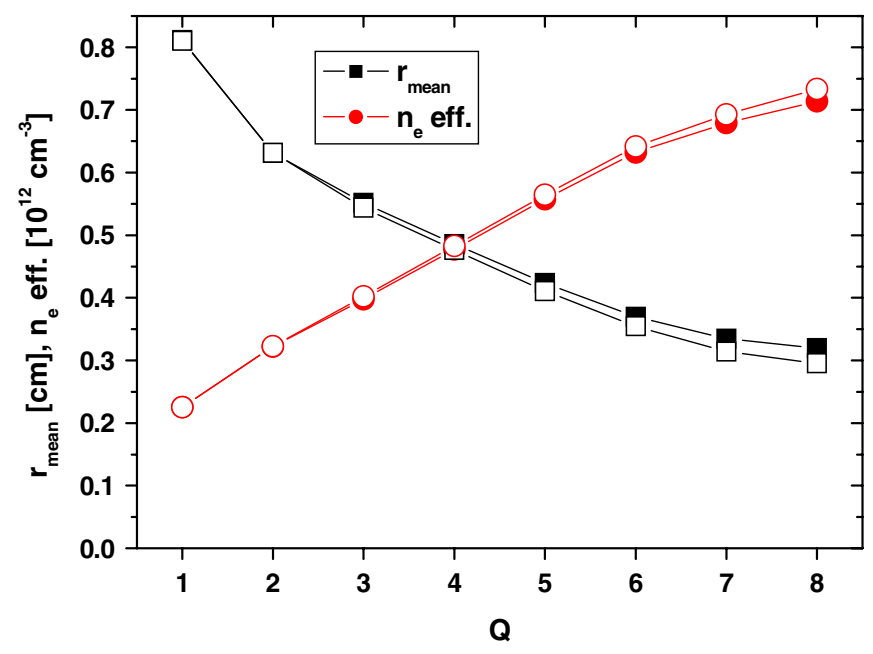

FIG. 12. (Color) Mean radius of the ion beam at the plasma electrode (squares) and effective electron density (circles) for the neon ions with different charge states. Open symbols are for the ${ }^{22} \mathrm{Ne}$ ions, solid ones are for ${ }^{20} \mathrm{Ne}$.

It is experimentally found that the output of highly charged ions can be increased when a lighter gas is mixed into the plasma [3]. The reasons for this so-called gasmixing effect are not clearly understood. Some authors explain the effect from the point of view of reduction of the ion heating rate due to the reduced mean charge state of ions in the plasma when the lighter gas is present [23]; a different explanation is based on the increased evaporative cooling rate due to transfer of energy from the heavier ions to the lighter ones [24].

We have studied the gas-mixing effect for argon and neon in simulations where these gases are mixed with helium/oxygen in different proportions. The results of these simulations were compared with simulations of $\mathrm{Ar} / \mathrm{Ne}$ plasmas having the same particle statistical weights and approximately the same total gas pressure. A positive gas-mixing effect was not observed. Ion temperatures are not significantly affected by addition of the lighter gases into the discharge, indicating small changes in the ion heating/cooling rate. We exclude therefore the ion cooling explanations for the gas-mixing effect, at least in the zero potential dip approximation. From the above considerations we conclude that the physical mechanisms for the isotope anomaly and for the gas-mixing effect are different.

\section{G. Wall-coating effect}

It is known that the performance of ECR ion sources is strongly affected by the wall conditions in the source. In particular, the extracted currents of highly charged ions increase noticeably when the walls are covered with thin films of oxides. This so-called wall-coating effect [3] is usually explained in terms of higher secondary electron emission from oxidized surfaces resulting in a lower plasma potential.
As an alternative (complementary) approach we have studied with our code the influence of the wall conditions on the energy and angular distributions of the fast backscattered atoms that are created after wall neutralization of ions. Indeed, before the ions are lost at the walls they are accelerated in the plasma sheath to a relatively high energy, proportional to the plasma potential (typical experimental values of the plasma potential are a few tens of Volts) and the charge state of the ion. The direction of this acceleration is along the surface normal. For ion energies in the range of a few tens of $\mathrm{eV}$, the rebouncing atoms retain up to $5 \%$ of the primary ion energy depending on the wall material, surface conditions, ion mass, and other factors [14]. This results in the creation of fluxes of relatively energetic (in the range of a few $\mathrm{eV}$ ) atoms, which in turn leads to extra heating of the ion population after ionization of these atoms.

Reflection of atoms from the surface can be described as mainly a combination of diffusive and specular scattering. The diffusive reflection is considered to result from multiple scattering from a rough surface or from the layers directly beneath the surface. The angular distribution of these scattered atoms is given by Lambert's cosine law. The specular reflection results from single collisions between the impinging ion and solid atoms. Because the impinging ions are directed along the surface normal, the specularly reflected atoms will rebounce from the surface in the normal direction.

Another contribution to the fluxes of atoms after bombardment of a surface with ions is the ion-induced resputtering of gases that are trapped in the wall material. The energy distribution of the desorbed atoms can be described as consisting of two components, i.e., a slow component with a temperature that corresponds to the wall temperature and having a cosinelike angular distribution, and a fast component with a Maxwell-Boltzmann distribution at high temperature (a few eV). Its angular distribution shows strong peaking along the surface normal [25].

We studied the influence of fast atoms assuming that they have a single Maxwell-Boltzmann energy distribution and neglecting the possible variations in the temperature for different charge states of the primary ions. Plasma parameters are then calculated for temperatures of the rebouncing atoms varying from $0.025 \mathrm{eV}$ (wall temperature) to $10 \mathrm{eV}$.

Simulations show that the temperature of the rebouncing atoms has only a small effect on plasma behavior when the scattering angles of the atoms are defined according the "cosine law." The atoms are scattered and cooled at the walls many times before they are ionized and trapped in the plasma and then transfer their energy to the ion population.

The situation is quite different when we assume instead that all neutralized ions are back reflected at normal angles to the wall. These atoms are then effectively ionized before being thermalized in collisions with the walls. The result- 


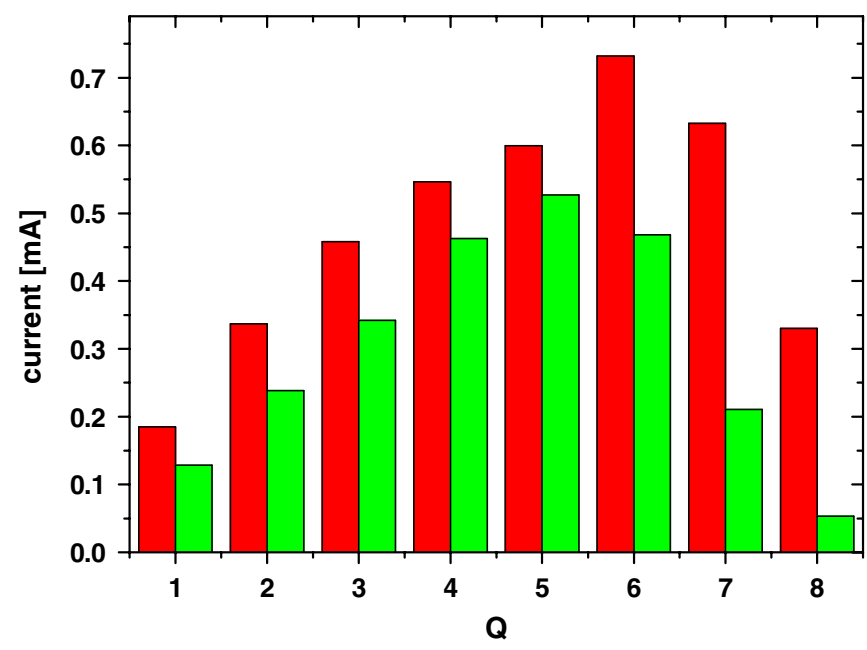

FIG. 13. (Color) Charge-state distributions of the extracted neon ions for the reference conditions (green columns) and for the doubled plasma chamber length (red columns).

ing ion heating leads to a loss of confinement. It is observed that the ion output decreases drastically if the energy of the scattered atoms is above a few $\mathrm{eV}$.

It is rather difficult to estimate realistic distributions of scattering angles and energies of the backscattered atoms inside the source. The general conclusion from our simulations is that diffusive scattering from the walls is more preferable for optimizing source performance. One way to control the fast atom distributions is the use of oxidized surfaces. These are known to have higher energy absorption coefficients and a higher scattering ability, resulting in a decrease of the gas temperature inside the source.

\section{H. Source length scaling}

Preliminary studies have been performed on the effect of doubling the plasma chamber length on source performance. We have used the same solenoidal magnetic field as in the reference conditions, but decreased the radial component by a factor of 2 in order to respect Maxwell's equations. The hexapolar field was not changed. To keep the gas pressure at approximately the same level as before, the statistical weight of the particles was doubled. The charge-state distribution of the extracted neon ions is plotted in Fig. 13 together with the CSD for the original chamber length. The increase in the currents of the highest charge states of the neon ions is remarkable. Simultaneously, longer ion confinement times are observed, confirming that these times are proportional to the plasma length. We conclude that long plasma chamber lengths are very beneficial for maximizing source performance with respect to charge states and currents.

\section{CONCLUSION}

The results of our simulations clearly show the importance of taking into account the diffusion processes in the
ECRIS plasma when modeling ion dynamics. The extracted ion currents and shape of the plasma are well reproduced by the code. Our simulations do not include the existence of electric fields that confine the ions in the plasma, but such effects are probably of minor importance, at least at relatively high gas pressure. It is shown that the long-standing problem of the isotope anomaly is connected to the ion diffusion dynamics in the plasma. The importance of taking into consideration the pressure gradients inside the plasma and parameters of the fast backscattered atoms is confirmed by the code.

The model can be used for studies of various effects in ECRIS plasmas, such as scaling of the source dimensions and of the magnetic field structure. Predictions of the beam shapes of the extracted ions can be made. Application of the model to simulations of charge breeding in on-line ECR ion sources is feasible and requires only small modifications of the code.

To improve the code and to reduce the present model dependence, the most important step is to incorporate the electron dynamics into the code, including the rf electron heating to obtain a more realistic description of the electron population parameters. This is planned to be done in the future.

\section{ACKNOWLEDGMENTS}

This work is part of the research program of the "Stichting voor Fundamenteel Onderzoek der Materie (FOM)," which is financially supported by the "Nederlandse Organisatie voor Wetenschappelijk Onderzoek (NWO)." Also, it was supported by the European Commission within the Sixth Framework Programme through I3-EURONS (Contract No. RII3-CT2004-506065), by the University of Groningen, and the Gesellschaft für Schwerionenforschung $\mathrm{mbH}$ (GSI), Darmstadt.

[1] R. Geller, Electron Cyclotron Resonance Ion Sources and ECR Plasma (Institute of Physics, Bristol, 1996).

[2] D. Hitz, A. Girard, G. Melin, S. Gammino, G. Ciavola, and L. Celona, Rev. Sci. Instrum. 73, 509 (2002).

[3] A. G. Drentje, Rev. Sci. Instrum. 74, 2631 (2003), and references therein.

[4] G. Douysset, H. Khodja, A. Girard, and J. P. Briand, Phys. Rev. E 61, 3015 (2000).

[5] V. Mironov, S. Runkel, and K. E. Stiebing, Rev. Sci. Instrum. 72, 2271 (2001).

[6] H. R. Kremers, J. P. M. Beijers, and S. Brandenburg, Rev. Sci. Instrum. 77, 03A311 (2006).

[7] http://laacg1.lanl.gov/laacg.

[8] K. Halbach, Nucl. Instrum. Methods 169, 1 (1980).

[9] J.P. Verboncoeur, A. B. Langdon, and N.T. Gladd, Comput. Phys. Commun. 87, 199 (1995).

[10] K. Nanbu, Phys. Rev. E 55, 4642 (1997).

[11] T. Takizuka and H. Abe, J. Comput. Phys. 25, 205 (1977). 
[12] P. Mazzotta, G. Mazzitelli, S. Colfrancesco, and N. Vittorio, Astron. Astrophys. Suppl. Ser. 133, 403 (1998).

[13] J. Greenwood, Vacuum 67, 217 (2002).

[14] H. F. Winters, H. Coufal, C. T. Rettner, and D. S. Bethune, Phys. Rev. B 41, 6240 (1990).

[15] F. O. Goodman and H. Y. Wachman, J. Chem. Phys. 46, 2376 (1967).

[16] J. D. Huba, NRL Plasma Formulary Report No. NRL/PU/ 6790-04-477, 2004.

[17] D. Wutte, S. Abbott, M. A. Leitner, and C. M. Lyneis, Rev. Sci. Instrum. 73, 521 (2002).

[18] D. Leitner and C. M. Lyneis, in Proceedings of the 21st Particle Accelerator Conference, Knoxville, 2005 (IEEE, Piscataway, NJ, 2005).

[19] D. S. Todd, D. Leitner, M. Leitner, C. M. Lyneis, and J.
Qiang, Rev. Sci. Instrum. 77, 03A338 (2006).

[20] A. G. Drentje, A. Girard, D. Hitz, and G. Melin, Rev. Sci. Instrum. 71, 623 (2000).

[21] O. Tarvainen, P. Suominen, T. Ropponen, T. Kalvas, P. Heikkinen, and H. Koivisto, Rev. Sci. Instrum. 76, 093304 (2005).

[22] T. D. Rognlien and T. A. Cutler, Nucl. Fusion 20, 1003 (1980).

[23] R. Geller, in Proceedings of the International Conference on ECR Ion Sources and their Applications, East Lansing, 1987, edited by J. Parker (NSCL Report No. MCUCP-47), p. 15.

[24] G. Shirkov, Plasma Sources Sci. Technol. 2, 250 (1993).

[25] H. Feil, J. van Zwol, S. T. de Zwart, J. Dielman, and B. J. Garrison, Phys. Rev. B 43, 13695 (1991). 